\title{
Mittag-Leffler fonksiyonunu içeren analitik fonksiyonların bazı özellikleri
}

\author{
Some properties of analytic functions involving the Mittag-Leffler function
}

\author{
Asena ÇETINKAYA*1,a ${ }^{* y a}$ MERT $^{2, b}$ \\ ${ }^{1}$ İstanbul Kültür Üniversitesi, Matematik ve Bilgisayar Bilimleri Bölümü, 34158, İstanbul, Türkiye \\ ${ }^{2}$ Tekirdă̆ Namık Kemal Üniversitesi, Matematik Bölümü, 59030, Tekirdağ, Türkiye
}

• Geliş tarihi / Received: 19.01.2021 • • Düzeltilerek geliş tarihi / Received in revised form: 16.02.2021 • Kabul tarihi / Accepted: 23.02.2021

$\ddot{O} z$

Mittag-Leffler fonksiyonu 1903 yılında İsveçli matematikçi Magnus Gustav Mittag-Leffler tarafından tanımlanmıştır. Daha sonra, araştırmacılar farklı parametreler ilave ederek bu fonksiyonu genelleştirmiştir. 2015 yılında, Bansal ve Prajabat, Mittag-Leffler fonksiyonunu normalize etmiş ve bu fonksiyonun açık birim diskte yalınkatlık, yıldızıllık, konvekslik ve konvekse yakınlık gibi belirli geometrik özelliklere sahip olduğunu gösteren yeterli koşullar elde etmiş̧tir. $\mathrm{Bu}$ araştırma makalesinden sonra, Mittag-Leffler fonksiyonu yalınkat fonksiyonlar teorisi çalışmalarında popüler olmuştur. Bu güncel çalışmada, $S_{\alpha, \beta}^{\gamma}(k, A, B)$ ile gösterilen Mittag-Leffler fonksiyonunu içeren analitik fonksiyonların yeni bir sınıfı tanımlanmıştır. Ayrıca, bu fonksiyon sınıfının negatif katsayıları içeren bir alt sınıfı da tanımlanmıştır. Bu fonksiyon sınıfı için katsayı tahminleri, büyüme ve distorsiyon teoremleri elde edilmiştir. Bununla birlikte, bu sınıf için integral eşitsizlikleri de elde edilmiştir. Ayrıca parametrelerin özel değerleri için, bu makalede tanımlanan sınıfların, araştırmacılar tarafından tanımlanan bazı fonksiyon sınıflarına indirgendiği sonucuna varılmıştır.

Anahtar kelimeler: Analitik fonksiyonlar, Katsayı eşitsizliği, Yıldızıl fonksiyonlar

\begin{abstract}
The Mittag-Leffler function was defined by Swedish mathematican Magnus Gustav Mittag-Leffler in 1903. Later, researchers generalized this function by including different parameters. In 2015, Bansal and Prajabat normalized the Mittag-Leffler function and get several sufficient conditions so that the Mittag-Leffler function has certain geometric properties such as univalency, starlikeness, convexity and close-to-convexity in the open unit disc. After this research paper, the Mittag-Leffler function became popular in the studies of univalent functions theory. In this current study, we define a new class of analytic functions involving the Mittag-Leffler function denoted by $S_{\alpha, \beta}^{\gamma}(k, A, B)$. We also introduce a subclass of this function class, which is involving negative coefficients. We introduce coefficient estimates, growth and distortion theorems for this function class. Moreover, we obtain integral mean inequalities for this class. We also conclude that for special values of parameters, the classes introduced in this paper are reduced to the several function classes which are defined by researchers.
\end{abstract}

Keywords: Analytic functions, Coefficient inequality, Starlike functions

\footnotetext{
*a Asena ÇETINKAYA; asnfigen@hotmail.com, Tel: (0212) 4984141, orcid.org/0000-0002-8815-5642

${ }^{\mathrm{b}}$ orcid.org/0000-0002-8791-3341
} 


\section{Giriş}

$\mathbb{C}$ kompleks düzlem olmak üzere $\mathbb{D}=\{z: z \in$ $\mathbb{C}$ ve $|z|<1\}$ açık birim disk olarak tanımlanır. $D \subset \mathbb{C}$ kompleks düzlemde basit bağlantılı bir bölge olmak üzere $f: D \rightarrow \mathbb{C}$ fonksiyonu bire-bir ise $f$ fonksiyonuna yalınkat fonksiyon denir. $\mathbb{D}$ açık birim diskinde analitik ve $f(0)=0$ ve $f^{\prime}(0)=1$ koşullarını sağlayan $f$ fonksiyonuna normalize edilmiş analitik fonksiyon denir. $\mathbb{D}$ açık birim diskinde normalize edilmiş analitik fonksiyonların sınıfı $\mathcal{A}$ ile gösterilir ve her $f \in \mathcal{A}$ fonksiyonu

$f(z)=z+\sum_{n=2}^{\infty} a_{n} z^{n}$

şeklindeki Taylor seri açılımına sahiptir. $\mathbb{D}$ diskinde yalınkat ve $\mathcal{A}$ sınıfının altkümesi olan fonksiyonlar sınıfı $S$ ile gösterilir. Basit bağlantılı bir $D \subset \mathbb{C}$ bölgesinde bir $z_{0}$ noktası verilsin. $z_{0}$ noktasını her $z \in D$ noktasına birleştiren doğru parçası $D$ bölgesinin sınırını yalnız bir noktada kesiyorsa $D$ bölgesine $z_{0}$ noktasına göre yıldızıl bölge denir. Eğer $z_{0}$ noktasi orjinde ise bu bölgeye orjine göre yıldızıl bölge denir. $S$ sinıfının bir alt sınıfi olan ve görüntü bölgesi orjine göre yıldızıl bölge olan fonksiyonlara yıldızıl fonksiyonlar denir. Yıldızıl fonksiyonlar sınıfı $S^{*}$ ile gösterilir. Analitik olarak, $f \in S^{*}$ olması için gerek ve yeter şart her $z \in \mathbb{D}$ için
$\operatorname{Re}\left(\frac{z f^{\prime}(z)}{f(z)}\right)>0$

eşitsizliğinin sağlanmasıdır.

Goodman (1991) yaptığı çalışmalarda $f$ fonksiyonunun $\mathbb{D}$ açık birim diskinde bulunan $\xi \in \mathbb{D}$ merkezli her dairesel yayı yıldızıl bir yay üzerine resmettiğini göstermiştir. Bu özellikteki bir $f \in \mathcal{A}$ fonksiyonunu düzgün yıldızıl fonksiyon olarak isimlendirmiştir. $S$ sınıfının bir altsınıfı olan düzgün yıldızıl fonksiyonlar sınıfı $U S$ ile gösterilmiştir. Ronning (1993) düzgün yıldızıl fonksiyonlar sınıfını kapsamlı bir şekilde çalışmıştır. Daha sonra, Kanas ve Wisniowska (2000) $k$-US ile gösterilen $k$-düzgün yıldızıl fonksiyonlar sınıfını tanımlamıştır. Bu sınıfa ait $f \in \mathcal{A}$ fonksiyonları

$\operatorname{Re}\left(\frac{z f^{\prime}(z)}{f(z)}\right)>k\left|\frac{z f^{\prime}(z)}{f(z)}-1\right| \quad(k \geq 0 ; z \in \mathbb{D})$

analitik kriterini sağlamaktadır. $k=1$ özel durumu için $1-U S \equiv U S$ sinıfi elde edilir. Ayrıca, $k=0$ için $0-U S \equiv S^{*}$ olduğu açıktır.

Bharati vd., (1997) $\quad k$-düzgün yıldızıl fonksiyonlar sınıfinı genelleştirmiş ve $k-$ $U S(\delta)$ ile gösterilen $\delta$ mertebeden $k$-düzgün yıldızıl fonksiyonlar sınıfını tanımlamıştır. Bu sınıf Tanım 1.1 ile ifade edilmiştir.

Tanım 1.1. $f$ fonksiyonu (1) formunda tanımlı bir fonksiyon olmak üzere

$R e\left(\frac{z f^{\prime}(z)}{f(z)}\right)>k\left|\frac{z f^{\prime}(z)}{f(z)}-1\right|+\delta \quad(0 \leq \delta<1 ; k \geq 0 ; z \in \mathbb{D})$

analitik kriterini sağlıyorsa $k-U S(\delta)$ sınıfındadır denir. Özellikle, $k-U S(0) \equiv k-U S$ olduğu açıktır.

$\mathbb{D}$ diskinde pozitif reel kısma sahip, analitik ve $p(0)=1$ koşulunu sağlayan $p$ fonksiyonlarının sınıfı $\mathcal{P}$ ile gösterilmektedir. Bu sınıfa Caratheodory sınıfı denir. $\mathbb{D}$ diskinde analitik, her $z \in \mathbb{D}$ için $w(0)=0$ ve $|w(z)|<1$ koşullarını sağlayan $w$ fonksiyonlarının sınıfı $\Omega$ ile gösterilmektedir. Bu sınıfa ait fonksiyonlar Schwarz fonksiyonu olarak adlandırılır. $f_{1}$ ve $f_{2}$ fonksiyonları $\mathbb{D}$ diskinde analitik fonksiyonlar olmak üzere, her $z \in \mathbb{D}$ için $f_{1}(z)=f_{2}(w(z))$ olacak şekilde bir $w \in \Omega$ fonksiyonu varsa, $f_{1}$ fonksiyonu $f_{2}$ fonksiyonuna sabordinedir denir ve $f_{1} \prec f_{2}$ ile gösterilir (Duren, 1983).

$f_{1}(z)=z+\sum_{n=2}^{\infty} a_{n} z^{n}$ ve $f_{2}(z)=z+\sum_{n=2}^{\infty} b_{n} z^{n}$ fonksiyonları $\mathcal{A}$ sinıfının elemanları olmak üzere, bu fonksiyonların Hadamard çarpımı (veya konvolüsyonu)

$f_{1}(z) * f_{2}(z)=\left(f_{1} * f_{2}\right)(z)=z+\sum_{n=2}^{\infty} a_{n} b_{n} z^{n}$

ile gösterilir.

Janowski (1973) sabordinasyon koşullarını kullanarak $\mathcal{P}(A, B)$ sınıfını tanımlamıştır. $p(0)=1$ koşulunu sağlayan bir $p$ analitik fonksiyonunun $\mathcal{P}(A, B)$ sınıfina ait olması için gerek ve yeter koşul her $z \in \mathbb{D}$ için 
$p(z) \prec \frac{1+A z}{1+B z} \quad(-1 \leq B<A \leq 1)$

şartının sağlanmasıdır. Geometrik olarak, bir $p \in \mathcal{P}(A, B)$ fonksiyonu $\mathbb{D}$ diskini

$\psi(A, B):=\left\{w:\left|w-\frac{1-A B}{1-B^{2}}\right|<\frac{A-B}{1-B^{2}}\right\}$

ile tanımlı $\psi(A, B)$ bölgesine resmeder. Bu bölge, reel eksende çap uç noktaları $D_{1}=\frac{1-A}{1-B}$ ve $D_{2}=$ $\frac{1+A}{1+B}\left(0<D_{1}<1<D_{2}\right)$ olan açık bir dairesel diski temsil etmektedir.

1903 yılında İsveçli matematikçi Mittag-Leffler

$E_{\alpha}(z)=\sum_{n=0}^{\infty} \frac{z^{n}}{\Gamma(\alpha n+1)} \quad(\alpha \in \mathbb{C} ; \operatorname{Re}(\alpha)>0)$

fonksiyonunu tanımlamıştır (Mittag-Leffler, 1903). Burada $\Gamma$ (.) notasyonu Gama fonksiyonunu belirtir. $E_{\alpha}(z)$ fonksiyonu Wiman tarafindan

$E_{\alpha, \beta}(z)=\sum_{n=0}^{\infty} \frac{z^{n}}{\Gamma(\alpha n+\beta)} \quad(\alpha, \beta \in \mathbb{C} ; \operatorname{Re}(\alpha)>0 ; \operatorname{Re}(\beta)>0)$

olarak genelleştirilmiştir (Wiman, 1905). Daha sonra, Mittag-Leffler fonksiyonu ve onun çeşitli genelleştirmeleri kesirli diferansiyel denklemlerin, Levy uçuş problemlerinin ve diğer başka problemlerin çözümünde kullanılmıştır (Bansal ve Prajapat, 2016; Gorenflo vd., 2014).

1971 yılında Prabhakar, Mittag-Leffler fonksiyonunu genelleştirmiş ve

$E_{\alpha, \beta}^{\gamma}(z)=\sum_{n=0}^{\infty} \frac{(\gamma)_{n}}{\Gamma(\alpha n+\beta)} \frac{z^{n}}{n !} \quad(\alpha, \beta, \gamma \in \mathbb{C} ; \operatorname{Re}(\alpha)>0 ; \operatorname{Re}(\beta)>0 ; \operatorname{Re}(\gamma)>0)$

şeklinde tanımlamıştır (Prabhakar, 1971). Burada $(\gamma)_{n}$ ifadesi

$(\gamma)_{n}=\frac{\Gamma(\gamma+n)}{\Gamma(\gamma)}=\left\{\begin{array}{cc}1 & (n=0) \\ \gamma(\gamma+1) \ldots(\gamma+n-1) & (n \in \mathbb{N})\end{array}\right.$

ile verilen Pochhammer sembolünü temsil etmektedir. $E_{\alpha, \beta}^{\gamma}(z)$ fonksiyonu için

$E_{\alpha, 1}^{1}(z)=: E_{\alpha}(z)$ ve $E_{\alpha, \beta}^{1}(z)=: E_{\alpha, \beta}(z)$

özel durumları mevcuttur.

(2) ile verilen $E_{\alpha, \beta}^{\gamma}(z)$ fonksiyonu $\mathcal{A}$ sınıfına ait değildir. $E_{\alpha, \beta}^{\gamma}(z)$ fonksiyonunun $\mathcal{A}$ sınıfına ait olması için $M_{\alpha, \beta}^{\gamma}(z)=\Gamma(\beta) z E_{\alpha, \beta}^{\gamma}(z)=z+\sum_{n=1}^{\infty} \frac{\Gamma(\beta)(\gamma)_{n}}{\Gamma(\alpha n+\beta)} \frac{z^{n+1}}{n !}$

şeklinde normalize edilmiştir. Bu çalışmalardan yola çıkarak Raducanu, $H_{\alpha, \beta}^{\gamma} f: \mathcal{A} \rightarrow \mathcal{A}$ lineer operatörünü 
$H_{\alpha, \beta}^{\gamma} f(z)=M_{\alpha, \beta}^{\gamma}(z) * f(z)=z+\sum_{n=2}^{\infty} \frac{\Gamma(\beta)(\gamma)_{n-1}}{\Gamma(\alpha(n-1)+\beta)(n-1) !} a_{n} z^{n}$

şeklinde tanımlamıştır (Raducanu, 2017). Burada $H_{0, \beta}^{1} f(z)=f(z)$ olduğu açıktır.

\section{Materyal ve metot}

Sabordinasyon prensibi ve $H_{\alpha, \beta}^{\gamma} f$ operatörü kullanılarak, Mittag-Leffler fonksiyonunu içeren yalınkat fonksiyonların yeni bir sınıfı Tanım 2.1 ile verilmiştir.

Tanım 2.1. $-1 \leq B<A \leq 1, k \geq 0$ ve $z \in \mathbb{D}$ olmak üzere (1) formunda tanımlanan $f$ fonksiyonu

$\frac{z\left(H_{\alpha, \beta}^{\gamma} f(z)\right)^{\prime}}{H_{\alpha, \beta}^{\gamma} f(z)}-k\left|\frac{z\left(H_{\alpha, \beta}^{\gamma} f(z)\right)^{\prime}}{H_{\alpha, \beta}^{\gamma} f(z)}-1\right| \prec \frac{1+A z}{1+B z}$

analitik kriterini sağlıyorsa $S_{\alpha, \beta}^{\gamma}(k, A, B)$ sınıfındadır denir.

$A, B, \alpha, \beta, \gamma$ ve $k$ için özel değerler alınırsa, yalınkat fonksiyonların bazı bilinen alt sınıfları elde edilir:

1. $A=1-2 \delta, B=-1, \alpha=0$ ve $\gamma=1$ değerleri alındığında, $S_{\alpha, \beta}^{\gamma}(k, A, B)$ sinıfı $k-U S(\delta)$ sınıfına indirgenir (Bharati vd., 1997).

2. $A=1, B=-1, \alpha=0$ ve $\gamma=1$ değerleri alındığında, $S_{\alpha, \beta}^{\gamma}(k, A, B)$ sinıfı $k-U S$ sinıfına indirgenir (Kanas ve Wisniowska, 2000).

3. $k=0, \alpha=0$ ve $\gamma=1$ değerleri alındığında, $S_{\alpha, \beta}^{\gamma}(k, A, B)$ sınıfı $S^{*}(A, B)$ sınıfına indirgenir (Janowski, 1973).

Tanım 2.2. $f \in \mathcal{A}$ olmak üzere negatif katsayılara sahip

$f(z)=z-\sum_{\mathrm{n}=2}^{\infty} a_{n} \mathrm{z}^{\mathrm{n}}, a_{n} \geq 0$

fonksiyonlarının sınıfı $T$ ile gösterilir. Bu sınıf negatif katsayılı fonksiyonlar sınıfı olarak adlandırılır ve $S$ sınıfının bir alt sınıfıdır (Silverman, 1975). $T$ ve $S_{\alpha, \beta}^{\gamma}(k, A, B)$ sınıfları göz önüne alınarak, $S_{\alpha, \beta}^{\gamma}(k, A, B)$ sinıfinın bir alt sinıfı olan negatif katsayılı fonksiyonların sinıfı

$T S_{\alpha, \beta}^{\gamma}(k, A, B):=T \cap S_{\alpha, \beta}^{\gamma}(k, A, B)$

olarak tanımlanmıştır.

$A, B, \alpha, \beta, \gamma$ ve $k$ için özel değerler alınırsa, bazı bilinen alt sınıflar elde edilir:

1. $A=1-2 \delta, B=-1, \alpha=0$ ve $\gamma=1$ değerleri alındığında, $T S_{\alpha, \beta}^{\gamma}(k, A, B) \operatorname{sinıfı} k-T U S(\delta) \operatorname{sinıfina~}$ indirgenir (Bharati vd., 1997).

2. $A=1-2 \delta, B=-1, k=0, \alpha=0$ ve $\gamma=1$ değerleri alındığında, $T S_{\alpha, \beta}^{\gamma}(k, A, B)$ sinıfi $T S^{*}(\delta)$ sinıfina indirgenir (Silverman, 1975). 
Littlewood, sabordine olan iki analitik fonksiyonun arasındaki integral ilişkisini (Littlewood,1925) makalesinde ispatlamıştır. Bu ilişki Önerme 2.3 ile verilmiştir.

Önerme2.3. $f_{1}$ ve $f_{2}$ fonksiyonları $\mathbb{D}$ birim diskinde analitik olmak üzere $f_{1}(\mathrm{z}) \prec f_{2}(z)$ şartı sağlanıyorsa, $p>0$ ve $z=r e^{\theta}(0<r<1)$ için

$\int_{0}^{2 \pi}\left|f_{1}(z)\right|^{p} d \theta \leq \int_{0}^{2 \pi}\left|f_{2}(z)\right|^{p} d \theta$

integral eşitsizliği sağlanır.

Bu makalede, Mittag-Leffler fonksiyonunu içeren ve $S_{\alpha, \beta}^{\gamma}(k, A, B)$ ile gösterilen analitik fonksiyonların yeni bir sınıfı tanımlanmıştır. Bu yeni sınıfın, negatif katsayılı fonksiyonlara sahip ve $T S_{\alpha, \beta}^{\gamma}(k, A, B)$ ile gösterilen bir alt sınıfı da tanımlanmıştır. Bu sınıf için katsayı tahminleri, büyüme ve distorsiyon teoremleri elde edilmiştir. Ayrıca, Önerme 2.3. kullanılarak bu sınıfa ait ve sabordine olan iki analitik fonksiyon arasındaki integral eşitsizliği de verilmiştir.

\section{Bulgular}

Teorem 3.1.' de (1) formunda verilen bir $f$ fonksiyonunun $S_{\alpha, \beta}^{\gamma}(k, A, B)$ sınıfına ait olması için yeter koşul verilmiştir.

Teorem 3.1. (1) formunda tanımlanan $f$ fonksiyonu

$\sum_{n=2}^{\infty}\{(1+k(1+|B|))(n-1)+|B n-A|\} \Psi_{n}\left|a_{n}\right| \leq A-B$

eşitsizliğini sağlıyorsa $S_{\alpha, \beta}^{\gamma}(k, A, B)$ sınıfına aittir. Burada

$\Psi_{n}=\frac{\Gamma(\beta)(\gamma)_{n-1}}{\Gamma(\alpha(n-1)+\beta)(n-1) !}$.

İspat. (7) ile verilen eşitsizliğin sağlandığını varsayalım. Burada $f \in S_{\alpha, \beta}^{\gamma}(k, A, B)$ olduğunu göstereceğiz. Teoremi kanıtlamak için $\left|\frac{p(z)-1}{A-B p(z)}\right| \leq 1$ eşitsizliğinin sağlandığını göstermek yeterlidir. (4) ile verilen ifade göz önüne alınarak

$p(z)=\frac{z\left(H_{\alpha, \beta}^{\gamma} f(z)\right)^{\prime}}{H_{\alpha, \beta}^{\gamma} f(z)}-k\left|\frac{z\left(H_{\alpha, \beta}^{\gamma} f(z)\right)^{\prime}}{H_{\alpha, \beta}^{\gamma} f(z)}-1\right|$

olduğu görülür. Verilen eşitsizlik ve (3) ifadesi kullanılarak

$$
\begin{aligned}
\left|\frac{p(z)-1}{A-B p(z)}\right| & =\left|\frac{z\left(H_{\alpha, \beta}^{\gamma} f(z)\right)^{\prime}-H_{\alpha, \beta}^{\gamma} f(z)-k e^{i \theta}\left|z\left(H_{\alpha, \beta}^{\gamma} f(z)\right)^{\prime}-H_{\alpha, \beta}^{\gamma} f(z)\right|}{A H_{\alpha, \beta}^{\gamma} f(z)-B\left[z\left(H_{\alpha, \beta}^{\gamma} f(z)\right)^{\prime}-k e^{i \theta}\left|z\left(H_{\alpha, \beta}^{\gamma} f(z)\right)^{\prime}-H_{\alpha, \beta}^{\gamma} f(z)\right|\right]}\right| \\
& =\left|\frac{\sum_{n=2}^{\infty}(n-1) \Psi_{n} a_{n} z^{n}-k e^{i \theta}\left|\sum_{n=2}^{\infty}(n-1) \Psi_{n} a_{n} z^{n}\right|}{(A-B) z-\sum_{n=2}^{\infty}(B n-A) \Psi_{n} a_{n} z^{n}+B k e^{i \theta}\left|\sum_{n=2}^{\infty}(n-1) \Psi_{n} a_{n} z^{n}\right|}\right| \\
& \leq \frac{\sum_{n=2}^{\infty}(n-1) \Psi_{n}\left|a_{n}\right||z|^{n}+k \sum_{n=2}^{\infty}(n-1) \Psi_{n}\left|a_{n}\right||z|^{n}}{(A-B)|z|-\sum_{n=2}^{\infty}|B n-A| \Psi_{n}\left|a_{n}\right||z|^{n}-k|B| \sum_{n=2}^{\infty}(n-1) \Psi_{n}\left|a_{n}\right||z|^{n}}
\end{aligned}
$$




$$
\leq \frac{\sum_{n=2}^{\infty}(n-1)(1+k) \Psi_{n}\left|a_{n}\right|}{(A-B)-\sum_{n=2}^{\infty}|B n-A| \Psi_{n}\left|a_{n}\right|-k|B| \sum_{n=2}^{\infty}(n-1) \Psi_{n}\left|a_{n}\right|}
$$

elde edilir. Eşitsizlikte son satır üstten 1 ile sınırlıdır. Buradan yola çıkarak

$\sum_{n=2}^{\infty}\{(1+k(1+|B|))(n-1)+|B n-A|\} \Psi_{n}\left|a_{n}\right| \leq A-B$

elde edilir. Bu da $f \in S_{\alpha, \beta}^{\gamma}(k, A, B)$ olduğunu gösterir. Böylece ispat tamamlanır.

Teorem 3.2.' de (7) ile verilen eşitsizliğin (5) formundaki $f$ fonksiyonlarının $T S_{\alpha, \beta}^{\gamma}(k, A, B)$ sınıfına ait olması için gerek koşul olduğu da gösterilmiştir.

Teorem 3.2. $f \in T$ olsun. O halde, $f \in T S_{\alpha, \beta}^{\gamma}(k, A, B)$ olması için gerek ve yeter koşul

$\sum_{n=2}^{\infty}\{(1+k(1+|B|))(n-1)+|B n-A|\} \Psi_{n} a_{n} \leq A-B$

eşitsizliğinin sağlanmasıdır. Burada $\Psi_{n}$ değeri (8) denklemi ile verilir.

İspat. Teorem 3.1 göz önüne alınarak, Teorem 3.2' nin ispatında gerek koşulu vermek yeterli olacaktır. $T S_{\alpha, \beta}^{\gamma}(k, A, B) \subset S_{\alpha, \beta}^{\gamma}(k, A, B)$ olduğundan $f \in T S_{\alpha, \beta}^{\gamma}(k, A, B)$ fonksiyonları için

$p(z)=\frac{z\left(H_{\alpha, \beta}^{\gamma} f(z)\right)^{\prime}}{H_{\alpha, \beta}^{\gamma} f(z)}-k\left|\frac{z\left(H_{\alpha, \beta}^{\gamma} f(z)\right)^{\prime}}{H_{\alpha, \beta}^{\gamma} f(z)}-1\right|$

ifadesi

$\left|\frac{p(z)-1}{A-B p(z)}\right| \leq 1$

eşitsizliğinde yerine yazılarak ve gerekli hesaplamalar yapılarak

$$
\begin{aligned}
& \left|\frac{p(z)-1}{A-B p(z)}\right|=\left|\frac{z\left(H_{\alpha, \beta}^{\gamma} f(z)\right)^{\prime}-H_{\alpha, \beta}^{\gamma} f(z)-k e^{i \theta}\left|z\left(H_{\alpha, \beta}^{\gamma} f(z)\right)^{\prime}-H_{\alpha, \beta}^{\gamma} f(z)\right|}{A H_{\alpha, \beta}^{\gamma} f(z)-B\left[z\left(H_{\alpha, \beta}^{\gamma} f(z)\right)^{\prime}-k e^{i \theta}\left|z\left(H_{\alpha, \beta}^{\gamma} f(z)\right)^{\prime}-H_{\alpha, \beta}^{\gamma} f(z)\right|\right]}\right| \\
& =\left|\frac{\sum_{n=2}^{\infty}(n-1) \Psi_{n} a_{n} z^{n}+k e^{i \theta}\left|\sum_{n=2}^{\infty}(n-1) \Psi_{n} a_{n} z^{n}\right|}{(A-B) z+\sum_{n=2}^{\infty}(B n-A) \Psi_{n} a_{n} z^{n}+B k e^{i \theta}\left|\sum_{n=2}^{\infty}(n-1) \Psi_{n} a_{n} z^{n}\right|}\right|
\end{aligned}
$$

elde edilir. $\operatorname{Re}(z) \leq|z|$ eşitsizliği kullanılarak

$\operatorname{Re}\left(\frac{\sum_{\mathrm{n}=2}^{\infty}(n-1) \Psi_{n} a_{n} z^{n}+k e^{i \theta}\left|\sum_{\mathrm{n}=2}^{\infty}(n-1) \Psi_{n} a_{n} z^{n}\right|}{(A-B) z+\sum_{\mathrm{n}=2}^{\infty}(B n-A) \Psi_{n} a_{n} z^{n}+B k e^{i \theta}\left|\sum_{\mathrm{n}=2}^{\infty}(n-1) \Psi_{n} a_{n} z^{n}\right|}\right) \leq 1$

elde edilir. $z$ reel sayı seçilerek ve $z \rightarrow 1^{-}$alınarak 
$\sum_{n=2}^{\infty}\{(1+k(1-B))(n-1)-(B n-A)\} \Psi_{n} a_{n} \leq A-B$

veya buna denk olarak

$\sum_{n=2}^{\infty}\{(1+k(1+|B|))(n-1)+|B n-A|\} \Psi_{n} a_{n} \leq A-B$

elde edilir. Böylece ispat tamamlanır

Sonuç 3.3. (5) formunda verilen $f$ fonksiyonu $T S_{\alpha, \beta}^{\gamma}(k, A, B)$ sınıfına ait olsun. O halde, her $n \geq 2$ için $a_{n} \leq \frac{A-B}{((1+k(1+|B|))(n-1)+|B n-A|) \Psi_{n}}$

sağlanır. Burada $\Psi_{n}$ ifadesi (8) ile verilmektedir. Bu sonuç

$f(z)=z-\frac{A-B}{((1+k(1+|B|))(n-1)+|B n-A|) \Psi_{n}} z^{n} \quad(n \geq 2)$

fonksiyonu için kesindir.

$T S_{\alpha, \beta}^{\gamma}(k, A, B)$ sınıfının büyüme ve distorsiyon teoremleri sırasıyla aşağıdaki gibi verilmiştir.

Teorem 3.4. (5) formunda verilen $f$ fonksiyonu $T S_{\alpha, \beta}^{\gamma}(k, A, B)$ sinifina ait olsun. O halde

$\Psi_{2}=\frac{\Gamma(\beta) \gamma}{\Gamma(\alpha+\beta)}$

olmak üzere

$|f(z)| \geq|z|-\frac{A-B}{((1+k(1+|B|))+|2 B-A|) \Psi_{2}}|z|^{2}$,

$|f(z)| \leq|z|+\frac{A-B}{((1+k(1+|B|))+|2 B-A|) \Psi_{2}}|z|^{2}$

eşitsizlikleri sağlanır. Bu eşitsizlikler kesindir.

İspat. Teorem 3.2.' de verilen (9) eşitsizliği göz önüne alınarak

$\varphi(n)=((1+k(1+|B|))(n-1)+|B n-A|) \Psi_{n}$

fonksiyonu seçilirse, $\varphi(n)(n \geq 2)$ fonksiyonunun artan bir fonksiyon olduğu görülür, çünkü

$\varphi(2) \sum_{n=2}^{\infty}\left|a_{n}\right| \leq \sum_{n=2}^{\infty} \varphi(n)\left|a_{n}\right| \leq A-B$

olduğu açiktır. Böylece 
$\sum_{n=2}^{\infty}\left|a_{n}\right| \leq \frac{A-B}{\varphi(2)}$

eşitsizliği yazılabilir. Buradan yola çıkarak

$$
\begin{aligned}
|f(z)| & \leq|z|+\sum_{n=2}^{\infty}\left|a_{n}\right||z|^{n} \leq|z|+|z|^{2} \sum_{n=2}^{\infty}\left|a_{n}\right| \\
& \leq|z|+\frac{A-B}{((1+k(1+|B|))+|2 B-A|) \Psi_{2}}|z|^{2}
\end{aligned}
$$

olduğu ispatlanır. Benzer şekilde

$$
\begin{aligned}
|f(z)| & \geq|z|-\sum_{n=2}^{\infty}\left|a_{n}\right||z|^{n} \geq|z|-|z|^{2} \sum_{n=2}^{\infty}\left|a_{n}\right| \\
& \geq|z|-\frac{A-B}{((1+k(1+|B|))+|2 B-A|) \Psi_{2}}|z|^{2}
\end{aligned}
$$

elde edilir. Bu sonuçlar $|z|=r$ ve $z=r e^{i(2 s+1) \pi}(s \in \mathbb{Z})$ olmak üzere

$$
f(z)=z-\frac{A-B}{((1+k(1+|B|))+|2 B-A|) \Psi_{2}} z^{2}
$$

fonksiyonu için kesindir.

Teorem 3.5. (5) formunda verilen $f$ fonksiyonu $T S_{\alpha, \beta}^{\gamma}(k, A, B)$ sinıfina ait olsun. O halde

$\left|f^{\prime}(z)\right| \geq 1-\frac{2(A-B)}{((1+k(1+|B|))+|2 B-A|) \Psi_{2}}|z|$

$\left|f^{\prime}(z)\right| \leq 1+\frac{2(A-B)}{((1+k(1+|B|))+|2 B-A|) \Psi_{2}}|z|$

eşitsizlikleri sağlanır. Bu eşitsizlikler kesindir.

İspat. Teorem 3.2. göz önüne alınarak

$\varphi(n)=((1+k(1+|B|))(n-1)+|B n-A|) \Psi_{n}$

fonksiyonu seçilirse, $\frac{\varphi(n)}{n}(n \geq 2)$ fonksiyonunun artan bir fonksiyon olduğu görülür, çünkü

$\frac{\varphi(2)}{2} \sum_{n=2}^{\infty} n\left|a_{n}\right| \leq \sum_{n=2}^{\infty} \frac{\varphi(n)}{n} n\left|a_{n}\right|=\sum_{n=2}^{\infty} \varphi(n)\left|a_{n}\right| \leq A-B$

olduğu açıktır. Böylece 
$\sum_{n=2}^{\infty} n\left|a_{n}\right| \leq \frac{2(A-B)}{\varphi(2)}$

elde edilir. Bu durumda

$\left|f^{\prime}(z)\right| \leq 1+|z| \sum_{n=2}^{\infty} n\left|a_{n}\right| \leq 1+\frac{2(A-B)}{((1+k(1+|B|))+|2 B-A|) \Psi_{2}}|z|$

bulunur. Benzer şekilde

$\left|f^{\prime}(z)\right| \geq 1-|z| \sum_{n=2}^{\infty} n\left|a_{n}\right| \geq 1-\frac{2(A-B)}{((1+k(1+|B|))+|2 B-A|) \Psi_{2}}|z|$

elde edilir. Bu sonuçlar (10) ile verilen fonksiyon için kesindir.

Bu bölümde son olarak, $T S_{\alpha, \beta}^{\gamma}(k, A, B)$ sınıfina ait sabordine olan iki analitik fonksiyonun arasındaki integral ilişkisi verilecektir.

Teorem 3.6. Farz edelim ki, $f \in T S_{\alpha, \beta}^{\gamma}(k, A, B), p>0,-1 \leq B<A \leq 1, k \geq 0$ olsun ve $g$ fonksiyonu

$g(z)=z-\frac{A-B}{((1+k(1+|B|))+|2 B-A|) \Psi_{2}} z^{2}$

ile tanımlansın. O halde, $z=r e^{\theta}(0<r<1)$ olmak üzere

$\int_{0}^{2 \pi}|f(z)|^{p} d \theta \leq \int_{0}^{2 \pi}|g(z)|^{p} d \theta$

integral eşitsizliği sağlanır.

İspat. (5) ile verilen

$f(z)=z-\sum_{n=2}^{\infty} a_{n} z^{n}, \quad a_{n} \geq 0$.

fonksiyonu ve $g$ fonksiyonu (6) bağıntısında yerine yazılarak

$\int_{0}^{2 \pi}\left|1-\sum_{n=2}^{\infty} a_{n} z^{n-1}\right|^{p} d \theta \leq \int_{0}^{2 \pi}\left|1-\frac{A-B}{((1+k(1+|B|))+|2 B-A|) \Psi_{2}} z\right|^{p} d \theta$

eşitsizliği elde edilir. Önerme 2.3 kullanılarak

$1-\sum_{n=2}^{\infty} a_{n} z^{n-1} \prec 1-\frac{A-B}{((1+k(1+|B|))+|2 B-A|) \Psi_{2}} z$

olduğunu ispatlamak yeterlidir. $w(0)=0$ ve $|w(z)|<1$ koşullarını sağlayan $w$ bir Schwarz fonksiyonu olmak üzere, yukarıdaki sabordinasyon 
$1-\sum_{n=2}^{\infty} a_{n} z^{n-1}=1-\frac{A-B}{((1+k(1+|B|))+|2 B-A|) \Psi_{2}} w(z)$

olarak yazılabilir. Buradan yola çıkarak

$$
\begin{aligned}
w(z) & =\left|\sum_{n=2}^{\infty} \frac{((1+k(1+|B|))+|2 B-A|) \Psi_{2}}{A-B} a_{n} z^{n-1}\right| \\
& \leq|z| \sum_{n=2}^{\infty} \frac{((1+k(1+|B|))+|2 B-A|) \Psi_{2}}{A-B} a_{n} \\
& \leq|z| \sum_{n=2}^{\infty} \frac{((1+k(1+|B|))(n-1)+|B n-A|) \Psi_{n}}{A-B} a_{n} \\
& \leq|z|<1
\end{aligned}
$$

elde edilir. Böylece ispat tamamlanır.

\section{Tartışma ve sonuçlar}

$\mathrm{Bu}$ makalede, Mittag-Leffler fonksiyonunu içeren yalınkat fonksiyonların yeni bir sinifi tanımlanmıştır. Ayrıca, bu sınıfın negatif katsayıları içeren bir alt sınıfı da tanımlanmıştır. $\mathrm{Bu}$ sınıf için katsayı tahminleri, büyüme ve distorsiyon teoremleri elde edilmiştir. Ayrıca, sabordine olan iki analitik fonksiyonun arasındaki integral ilişkisi de elde edilmişsir.

\section{Kaynaklar}

Bansal, D. and Prajapat, J. K. (2016). Certain geometric properties of the Mittag-Leffler functions. Complex Variables and Elliptic Equations, 61(3), 338-350.

https://doi.org/10.1080/17476933.2015.1079628

Bharati, R., Parvatham, R. and Swaminathan, A. (1997). On subclasses of uniformly convex functions and corresponding class of starlike functions. Tamkang Journal of Mathematics, 28(1), 17-32.

Duren, P. L. (1983). Univalent Functions. Springer, 259, XIV- 384.

Goodman, A.W. (1991). On Uniformly Starlike Functions. Journal of Mathematical Analysis and Applications, 155(2), 364-370.

Gorenflo, R., Kilbas, A.A., Mainardi, F. and Rogosin, S. (2014). Mittag-Leffler functions, related topics and applications. Springer, XIV- 443. https://doi.org/10.1007/978-3-662-43930-2

Janowski, W. (1973). Some Extremal problems for certain families of analytic functions I. Annales Polonici Mathematici, 28, 297-326. https://doi.org/10.4064/ap-28-3-297-326
Kanas, S. and Wisniowska, A. (2000). Conic domains and starlike functions. Revue Roumaine des Mathematiques Pures et Appliquees, 45(4), 647657.

Littlewood, J. E. (1925). On inequalities in the theory of functions. Proceedings of the London Mathematical Society, 23(2), 481-519. https://doi.org/10.1112/plms/s2-23.1.481

Mittag-Leffler, G. (1903). Sur la Nouvelle Fonction $\mathrm{E} \alpha(\mathrm{x})$. Comptes rendus de l'Académie des sciences Paris, 137, 554-558.

Prabhakar, T. R. (1971). A singular integral equation with a generalized Mittag-Leffler function in the Kernel. Yokohama Mathematical Journal, 19, 715.

Raducanu, D. (2017). Third-Order differential subordinations for analytic functions associated with generalized Mittag-Leffler functions. Mediterranean Journal of Mathematics, 14:167. https://doi.org.10.1007/s00009-017-0969-8

Rønning, F. (1993). Uniformly Convex functions and a corresponding class of starlike functions. Proceedings of the American Mathematical Society, 118(1), 189-196. https://doi.org/10.1090/S0002-9939-19931128729-7

Silverman, H. (1975). Univalent Functions with negative coefficients. Proceedings of the American Mathematical Society, 51, 109-116. https://doi.org/10.1090/S0002-9939-19750369678-0

Wiman, A. (1905). Über den fundamentalsatz in der teorie der funktionen $\mathrm{E} \_\mathrm{a}(\mathrm{x})$. Acta Mathematica, 29 , 191-201. https://doi.org.10.1007/BF02403202 